UCRL-JC-127998

PREPRINT

\title{
Sound Speed and Thermal Property Measurements of Inert Materials: Laser Spectroscopy and the Diamond-Anvil Cell
}

\author{
Joseph M. Zaug
}

This paper was prepared for submittal to the 1997 Topical Conference on Shock Compression of Condensed Matter of the American Physical Society Amherst, MA

July 27 - August 1, 1997

July 1997

This is a preprint of a paper intended for publication in a journal or proceedings. Since changes may be made before publication, this preprint is made available with the underotanding that it will not be cited or reproduced without the permisoion of the author. 


\section{DISCLAIMER}

This document was prepared as an account of work sponsored by an agency of the United States Government. Neither the United States Government nor the University of California nor any of their employees, makes any warranty, express or implied, or assumes any legal liability or responsibility for the accuracy, completeness, or usefulness of any information, apparatus, product, or process disclosed, or represents that its use would not infringe privately owned rights. Reference herein to any specific commercial product, process, or service by trade name, trademark, manufacturer, or otherwise, does not necessarily constitute or imply its endorsement, recommendation, or favoring by the United States Government or the University of California. The views and opinions of authors expressed herein do not necessarily state or reflect those of the United States Government or the University of California, and shall not be used for advertising or product endorsement purposes. 


\title{
SOUND SPEED AND THERMAL PROPERTYMEASUREMENTS OF INERT IMATERIALS: LASER SPECTROSCOPY AND THE DIAMOND-ANVIL CELL
}

\author{
Joseph M. Zaug \\ University of California, Lawrence Livermore National Laboratory \\ L-282, P.O. Box 808. Livermore, California 94550
}

\begin{abstract}
An indispensable companion to dynamical physics experimentation, static high-pressure diamond-anvil cell (DAC) research continues to evolve, with laser diagnostics, as an accurate and versatile experimental technique. Together, static and dynamic high-pressure and temperature physics and geophysical studies of deep planetary properties have bootstrapped each other in a process that has produced even higher pressures; consistently improved calibrations of temperature and pressures under static and dynamic conditions; and unprecedented data and understanding of materials, their elasticity, equations of state (EOS), and transport properties under extreme conditions. A collection of recent pressure and/or temperature dependent acoustic and thermal measurements and deduced mechanical properties and EOS data will be summarized for a wide range of materials including $\mathrm{H}_{2}, \mathrm{H}_{2} \mathrm{O}, \mathrm{H}_{2} \mathrm{~S}, \mathrm{D}_{2} \mathrm{~S}, \mathrm{CO}_{2}, \mathrm{CH}_{4}, \mathrm{~N}_{2} \mathrm{O}, \mathrm{CH}_{3} \mathrm{OH}$, $\mathrm{SiO}_{2}$, synthetic lubricants, PMMA, single crystal silicates and ceramic superconductors. Room $\mathrm{P} \& \mathrm{~T}$ sound speed measurements will be presented for the first time on single crystals of $\beta$-HMX. New highpressure and temperature diamond cell designs and pressure calibrant materials will be reviewed.
\end{abstract}

\section{INTRODUCTION}

The condition that we call "room pressure and temperature". (RPT) represents a minuscule fraction of the universe as we know it. In order to understand the physics and chemistry of non terrestrial environments, both natural and man made, requires the marriage of high pressure and temperature experimentation with theoretical and computational efforts. A good measure of this union lies in the physically-based predictive capabilities of computer models. Today, mantle convection, protein dynamics and detonation physics models rely heavily on empirically derived relations.

High pressure and temperature experiments currently fall in one of two categories: dynamic and static. Static high $P$ \& $T$ experiments serve to provide virtual snapshots of dynamic environments and therefore can elucidate unique effects of shock loading experiments. ${ }^{2}$ Shock dynamic and static DAC measurements compliment each other insofar as in their ability to completely map out the P-V-T space of candidate materials. DAC experiments are versatile in that one chooses a P-T point for measurements, while thermodynamically irteversible dynamic experiments can access the highest pressures and corresponding temperatures. The next generation of dynamic exper- iments will dramatically extend sample volumes to cubic centimeters (ATLAS at LANL) and push accessible pressures beyond one TPa (NIF at LLNL). The current $P$ $T$ regimes available to DAC's are shown superimposed over the geotherms of terrestrial and Jovian planets in Fig. 1.

DAC experiments have been helping to unravel fundamental problems: What are the physical and chemical mechanisms, including the kinetics, responsible for the onset of detonation? How does thermal transport, hot spots and fragmentation affect HE material sensitivity? What are the mechanisms responsible for reversible and irreversible protein denaturation or folding and what are their energetic pathways? Is Earth mantle convection a layered or continuum process? Aside from these programmatic issues, DAC research remains a vital avenue toward increasing our grasp of solid state physics and opening

\footnotetext{
1 For the sake of brevity, all references to static should be thought of as hydrostatic or quasi-hydrostatic

2 As an example, consider that the thermodynamic Grineisen parameter $\gamma$ decreases, for materials shocked to the fluid state. with increasing pressure in shock-wave experiments due to the increasing temperature along the Hugoniot $[1,2]$.
} 


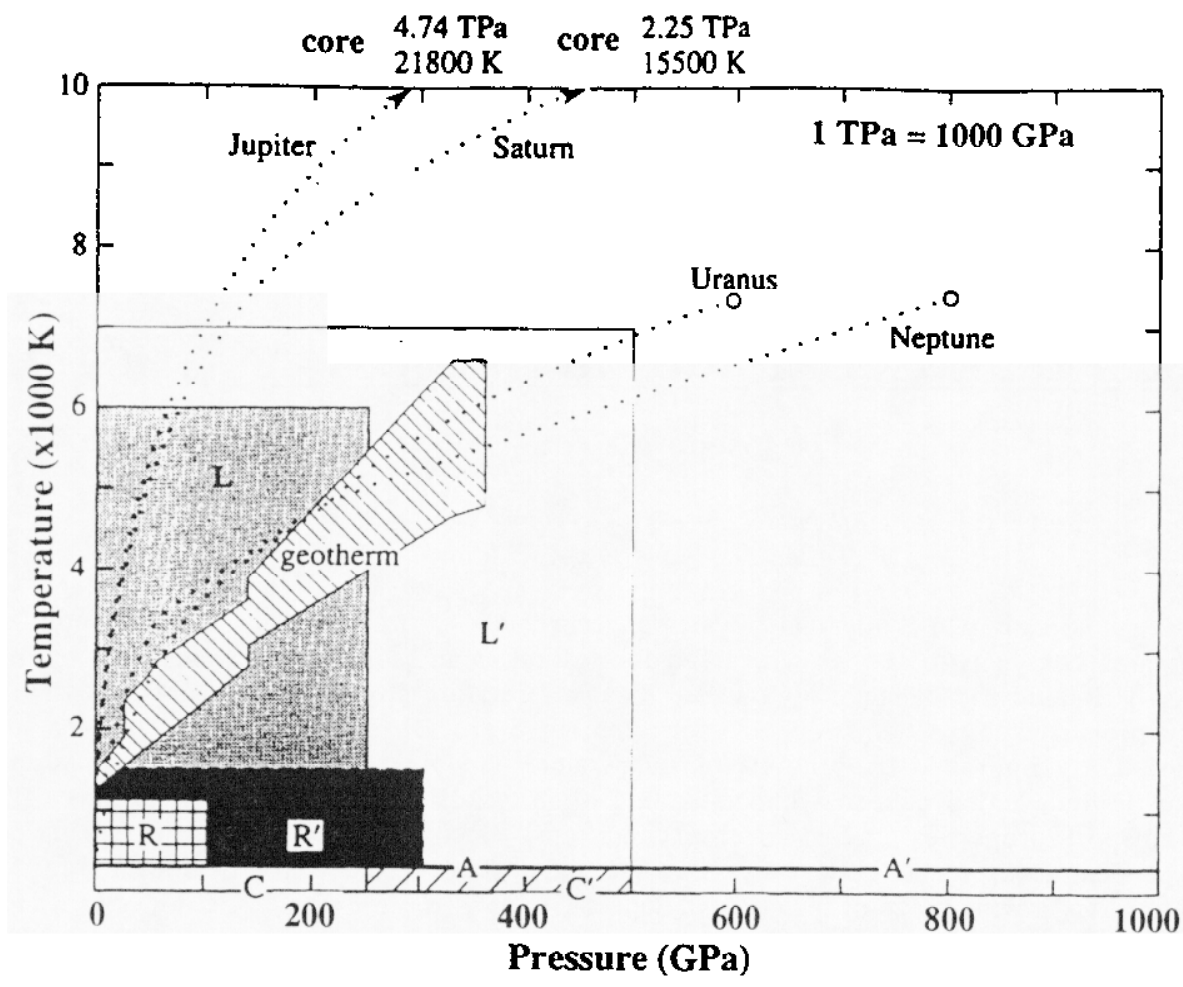

FIGURE 1. Presently accessible P-T ranges for DAC experiments [3]: A. ambient temperature; $C$ cryogenic; $R$, external resistance heating; $L$, laser heating. Corresponding ranges for future development are denored as $A^{\prime}, C^{\prime}, R^{\prime}$ and $L^{\prime}$. Dotted curves indicate estimated $P$. $T$ in Jovian planetary interiors [4] and the geotherm covers the temperature range in terrestrial planetary interiors [5].

our eyes to the experimentally wide open fields of liquid and glassy state physics.

This paper brings to light some recent DAC derived results from acoustic and thermal measurements. A focus on laser driven experiments serves to refine this review and should not detract from recent advances in traditional ultrasonic [6] and calorimetric [7] methods.

\section{ACOUSTIC \& THERMAL MEASUREMENTS IN THE DAC}

A brief description of the techniques used to make acoustic and thermal measurements is given below. Examples of these methods follow in the material studies section.

\section{Acoustic Measurements}

The acoustic measurements described here consist of classical, frequency-domain, optical Brillouin scattering (BS); and a time-domain method, impulsive stimulated light scattering (ISLS). ${ }^{1} \mathrm{~A}$ theoretical comparison between these two methods has been made elsewhere [8] and recently a direct inter-laboratory comparison has been made on San Carios olivine up to $17 \mathrm{GPa}$ [9]. Thermal DAC measurements employ either external heaters, external coolers, or lasers; or a combination of external and laser controlled systems. There are very few citations that describe simulataneous acoustic and thermal measurements in a DAC (i.e., $c(P, T))$.

Optical BS measurements represent the natural progression of the original technique [10]. In Brillowin spectroscopy the inelastic Bragg diffraction of incident photons from acoustic phonons, measured as frequency shifts $\Delta v$, allows one to determine longitudinal $\left(c_{1}\right)$ and transverse $\left(c_{t}\right)$ sound speeds.

$$
\Delta v_{x}= \pm 2 \frac{n c_{x}}{\lambda_{0}} \sin \left(\frac{\theta}{2}\right), \quad(\text { where } x=1 \text { or } t)
$$

In the above expression $\lambda_{0}$ is the incident light wave-

\footnotetext{
${ }^{1}$ For simplicity this generalized name is given, thereby making no assumptions of how the light is coupled to the material modes of a given sample.
} 
length, $n$ is the index of refraction and $\theta$ is the angle between the DAC axis (normal to the diamond table) and the incoming laser beam. For isotropic samples, where $\mathrm{n}=1$ at $\theta=90$, running concurrent BS experiments at two different scattering geometries provides a means to determine $n(P)$. Equation (1) is not valid in the presence of dispersion (i.e., where $\Delta v_{x} / \sin (\theta / 2)$ changes with $\theta$ at constant $\left.P \& T\right)$. If one studies anisotropic materials, and can determine the density, $\rho$, using $x$-ray diffraction (XRD), and can tolerate additional uncertainties in $c_{X}, n$ can be dropped from (1). Without XRD the density can be obtained by using a high pressure dilatometer [11]. If $n(P, T)$ is known the approximate density can be obtained by Lorentz-Lorenz's formula:

$$
\frac{4}{3} \pi N_{0} \alpha=\frac{M_{W}}{\rho} \frac{n^{2}-1}{n^{2}+2}
$$

where $\alpha$ is the molecular polarizability, and $N_{0}$ and $M_{w}$ are Avogadro's number and the molecular weight, respectively. ${ }^{\prime}$

Lastly, $\rho(P)$ can be calculated using

$$
\rho(P)-\rho_{0}=\int_{P_{0}}^{P} \frac{\gamma}{c^{2}} d P
$$

where $P_{0}, P_{0}$, and $\gamma$ are the RPT density, pressure and heat capacity ratio $C_{p} / C_{v}$. The bulk velocity is given by $c^{2}=c_{1}^{2}-(4 / 3) c_{t}^{2}$.

In the most general form of an ISLS experiment, two successive $1064 \mathrm{~nm}$ "excitation" pulses, $-80 \mathrm{ps}$ in duration, selected from the output train of a continuously pumped Q-switched and mode-locked Nd:YAG laser, are recombined in the sample volume of the DAC at an angle $\theta$, but otherwise coincident in space and time. When the polarizations of the two excitation pulses are parallel, interference establishes a periodic distribution of intensity in the sample and thus, in the case of an absorbing sample, a (spatially) periodic variation in the temperature and pressure which launches a set of three counterpropagating acoustic waves (one quasi-longitudinal and two quasi-transverse) of wavelength $\lambda_{A}$. The acoustic wavelength, in this case equal to the period of the optical grating, may be

1 Affects on $n$ by $\alpha(P)$ are on the order of $1: 10^{4}$ [12]. The polarizability in molecular crystals $\left(\mathrm{N}_{2} \mathrm{O}, \mathrm{CO}_{2}\right)$ drops by $-0.9 \%$ after $1 \mathrm{GPa}$ [13]. It is not clear that assumptions about $\alpha$ hold up say in high P-T salt solution studies. expressed in terms of the wavelength of the laser light $\lambda_{\mathrm{E}}$

$$
d=\lambda_{A}=\lambda_{E} /(2 \sin (\theta / 2)) .
$$

The impulsively excited acoustic waves induce a temporally and spatially periodic variation in the index of refraction of the sample. A third pulse, from the same Q-switched envelope as the excitation pulses is doubled to $532 \mathrm{~nm}$ and delayed by time of flight to generate the probe. Monitoring the intensity of the Bragg scattering of the probe by the acoustic grating as a function of probe delay serves to determine the frequency $\left(f_{A}\right)$, and hence the velocity $c=\left(f_{A} \cdot \lambda_{A}\right)$ of the acoustic waves. In general, the response of any Raman-active vibrational mode of the appropriate wavevector (whether acoustic or optical) to the periodic distribution of electric field generated by the crossed excitation pulses will be, if the period of vibration is long compared to the duration of the excitation pulses, a coherent standing wave which will serve to coherently scatter a delayed probe. For experiments reported here, the pulse duration is such that only acoustic modes are excited. In a DAC, the orientation of the acoustic grating with respect to the crystal axes may be varied by rotating the cell about an axis normal to the sample or diamond faces (See Fig. 2.). The velocity of sound, measured as a function of crystallographic direction (on 2-4 samples) and the density serves to determine the independent elastic constants

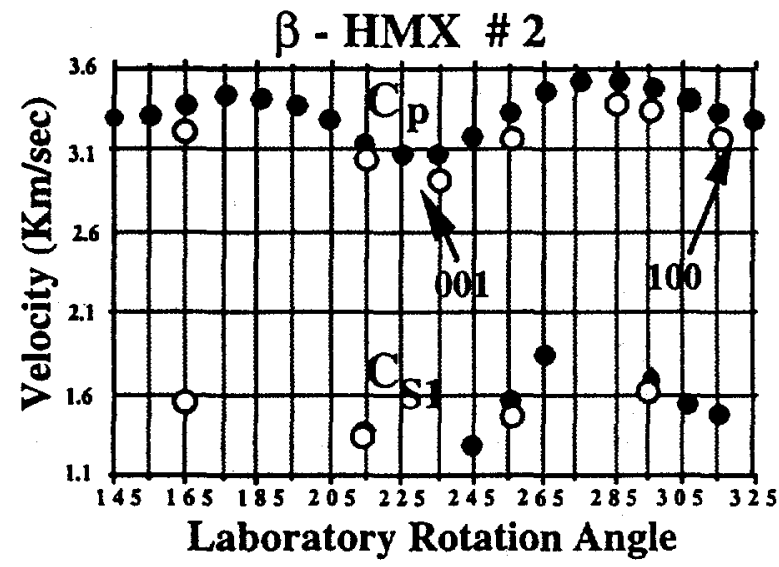

Figure 2. Quasi-longitudinal and quasi-shear sound speeds in single crystal $\beta$-HMX as a function of laboratory rotation angle and temperature. Open circles are $380^{\circ} \mathrm{K}$ and filled circles are at $297^{\circ} \mathrm{K}$. At the $322.5^{\circ}$ rotation angle ultrasonic propagation is most nearly along the crystallographic a axis.

\section{Thermal Measurement}

There are two distinct methods to apply heat to a sam- 
ple in a DAC: resistive heating (external and or internal) and internal laser heating. External resistive heating of DACs, in evacuated ovens, provides the most stable and accurate means of reaching increasingly better characterized pressures and temperatures up to $1700^{\circ} \mathrm{K}$. More care must be taken with internal (within the sample chamber) applications of heat. Because diamond is an excellent thermal conductor, internal resistive or ohmic DAC heating must be calibrated using known melting points of RPT ionic and metallic solids at several pressures. The resistivity of internal ohmic heating conductors and the thermal conductivity of deposited insulators will change with pressure and temperature. Unlike externally heated diamonds taken beyond $1400^{\circ} \mathrm{K}$, ohmically heated diamonds may survive beyond one high P-T cycle since the hottest portion of the diamond is within its stability field.

Laser heating can reach the highest static temperatures $\left(>6000^{\circ} \mathrm{K}\right)$, but not without encountering some rather vexing problems. Apparent sample temperature determinations (made from spectroradiometric interrogation of thin, laser heated, DAC samples) are dependent on the selection of conductivity models or temperature distributions that one inserts into a numerical expression for depth-averaged light intensities [14]. In addition, the magnitude of observed greybody emissivity (E) hinges on detailed thermal distributions of a sample which can vary with experimental configurations. It would seem that the large temperature gradients intrinsic to laser heating experiments (whether caused by the laser heating process, the measuring process, or a combination of both) is a non trivial issue due to the limited sample area and large variations in sample to pressure medium absorption ratios of the laser light in the DAC. To some degree radial temperature gradients have been reduced by using flat profile multimode lasers while axial temperature gradients have been reduced by simultaneous heating of both sides of the DAC [15]. The use of Raman scattering techniques to determine temperature may prove to be useful on laser heated samples that have Raman active modes [16].

In addition to acoustic studies, ISLS has been used to measure one dimensional thermal diffusivity [17]. As mentioned above, the spatial and temporal overlap of two $\sim 80$ ps $1064 \mathrm{~nm}$ pulses in a slightly absorbing sample results in a spatially periodic distribution of temperature where the period of the resultant grating, d, is given in (4). When the acoustic disturbance has been fully damped or has propagated beyond the area illuminated by the probe, a spatially periodic variation in the temperature and density remains. The characteristic time for the exponential decay of this "thermal" grating in a medium of density ( $p$ ) specific heat at constant pressure $\left(c_{p}\right)$ and thermal conductivity tensor $(\kappa)$ is given by

$$
\tau_{t h}=\frac{d^{2}}{4 \pi^{2} D_{t h}}
$$

where $d$ is the grating spacing and the thermal diffusivity is defined by $D_{t h}=\kappa / p C_{p}$. The grating spacing is kept sufficiently small so that conduction normal to the plane of the sample is unimportant (i.e., where one dimensional diffusion equation applies).

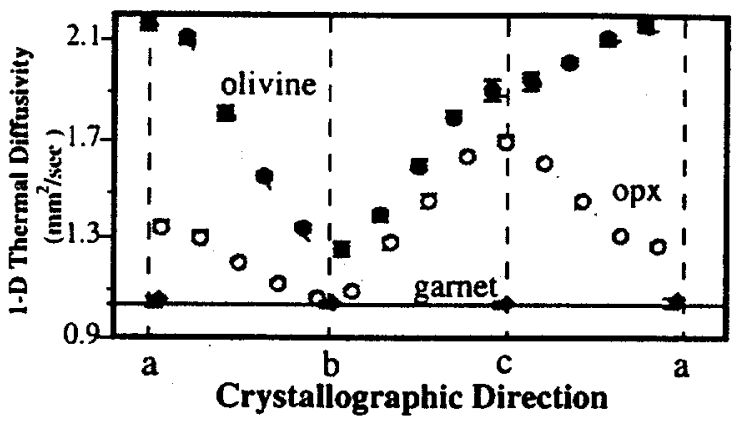

FIGURE 3. One-dimensional thermal diffusivity as a function of crystallographic direction in olivine, orthopyroxene and garnet at $298^{\circ} \mathrm{K}$ and 1 atm [17]. The b-a segment represents a $90^{\circ}$ rotation about the $c$ axis from $b$ to $a$, the b-c segment rotation is about the $a$ axis and the $a-c$ segment is about the $b$ axis. Uncertainties in fits to $D_{t h}\left(t_{t h}{ }^{-1}, d^{-2}\right)$ data are also provided.

\section{MATERIAL STUDIES}

Room pressure and temperature (gas to solid phase) materials have been investigated for their high pressure acoustic and thermal properties using the methods just described. As a result, determinations of the pressure and/or temperature dependence of structural and superconducting phase transitions, melting curves, elastic constants, acoustic damping rates, structural relaxation rates and amplitudes, anisotropic thermal diffusivity tensors, EOS's, and molecular polarizabilities have been made.

Planetary physics depends, to some degree, on the direct confrontation of theory and computational models with experimentally determined properties of candidate materials under relevant P-T conditions. The recent availability of elastic moduli on hydrogen (EOS up to $24 \mathrm{GPa}$ ) $[18,19]$, methane (up to $5 \mathrm{GPa}$ ) [20], nitrous oxide and carbon dioxide (up to $4.5 \mathrm{GPa}$ ) [21], water (ice VII) (2.2-7.4 GPa) [22], and $\beta-\mathrm{O}_{2}(6-9.5$ $\mathrm{GPa}$ [23]; including the pressure dependence of $\mathbf{n}$ and $\alpha$ in $\mathrm{N}_{2} \mathrm{O}$ and $\mathrm{CO}_{2}$; will improve our grasp of the compositional stratifications, oscillations and evolutional history of the Jovian planets and their moons.

The acoustic velocities, refractive index and elastic constants of two sulfides (hydrogen and deuterium) have been determined (up to $5 \mathrm{GPa}$ ) [24]. The results indicate 
that molecular rotation-translation coupling, though present at $1 \mathrm{GPa}$ (stronger in $\mathrm{D}_{2} \mathrm{~S}$ ), diminishes with increasing compression. This is probably due to the strengthening of hydrogen bonds in $\mathrm{H}_{2} \mathrm{~S} \& \mathrm{D}_{2} \mathrm{~S}$.

Traditionally the high pressure EOS has been the principle experimental probe in the regime of strong repulsive interactions. An acoustic and thermal study of the equilibrium and structural relaxation in methanol (up to $30.4 \mathrm{GPa}$ ) has demonstrated that the relaxation times may well provide more discrimination between model hamiltonians [25]. The relaxation times from this ISLS study were determined by direct measurement of the dispersion $c\left(f_{A}\right)$. where acoustic attenuation is observable, and by fitting the time-domain spectra to the form

$$
I=\left(A_{t h}-A_{a c} C(t) e^{-\gamma t} \cos \omega t+A_{s t r}\left(1-e^{-\gamma_{m} t}\right)\right)^{2}
$$

where $A_{t h}$ is the amplitude of the thermal grating. $A_{a c}$ the amplitude of the acoustic grating, $A_{s t r}$ is the amplitude from structural relaxation, $\omega=2 \pi f$ the circular frequency, $\gamma$ the temporal acoustic absorption coefficient, and $\tau_{\mathrm{m}}=1 / \gamma_{\mathrm{m}}$ the characteristic Mountain mode. $\mathrm{C}(\mathrm{t})$ is a geometric runout term where $t$ is the time delay of the probe beam.

Detonation physics is no different than planetary physics in the sense that there is legitimate need for experimental data. Mechanical and thermal transport data on appropriate single crystals of $\mathrm{HE}$ and binder materials, as a function of $P \& T$, is scarce. Recently the pressure dependence of the elastic constants (up to 0.30 $\mathrm{GPa}$ ) and density (up to $0.50 \mathrm{GPa}$ ) of poly (methyl methacrylate) were determined [26]. At $0.11 \mathrm{GPa}$ a second-order glass transition occurs resulting in a dramatic divergence in the bulk modulus from Young's modulus. Sound speed measurements in single crystals of $\beta$-HMX have been made as a function of temperature [27]. Preliminary one bar measurements indicate the quasi-longitudinal sound speeds $\left(c_{p}\right)$ from $298-380^{\circ} \mathrm{K}$, vary depending on the crystallographic propagation direction, from -3.5 to $-5.1 \%\left[\left(\mathrm{dc}_{\mathrm{p}} / \mathrm{dT}\right)_{\mathrm{p}}=-1.4 \mathrm{e}^{-3}\right.$ to $-2.2 e^{-3}$ ] which corresponds to a 7 to $10 \%$ drop in the adiabatic modulus as defined by $K_{s}=\rho c^{2}$. Similarly, the variation in the quasi-shear sound speeds $\left(c_{s}\right)$ is -2.2 to $-6.7 \%\left[\left(\mathrm{dc}_{\mathrm{s}} / \mathrm{dT}\right)_{\mathrm{p}}=-3.7 \mathrm{e}^{-4}\right.$ to $\left.-1.3 \mathrm{e}^{-3}\right]$ which corresponds to a 5 to $13 \%$ drop in primarily off diagonal contributions to the overall modulus. At one bar there is little or no hysteresis in quasi-longitudinal or quasishear sound speeds when cycling between 298 and 380 ${ }^{\circ} \mathrm{K}$.

Results from thermal DAC measurements on ammonium dinitramide map out the pressure, and temperature phase diagram, including a new polymorph transition, up to $10 \mathrm{GPa}$ from 198 - $393^{\circ} \mathrm{K}$ [28]. A novel DAC technique, high pressure \& low temperature matrix isolation, has been developed to trap intermediate (thermally initiated) product species from a thermally initiated propellant HNIW [29]. The method quenches the reaction and allows for spectroscopic determination of the trapped constituents.

Physical properties of lubricants determine the level of elastohydrodynamic lubrication (and hence mechanical wear) of gears. ball bearings and traction devices. Pressure dependent sound speed measurements were made for several paraffinic and naphthenic synthetic lubricants (up to $2.7 \mathrm{GPa}$ ) [30.31]. The slope $\mathrm{dc} / \mathrm{dP}$, in some of these lubricants, decreased remarkably at about $0.4 \mathrm{GPa}$. This change in slope could be an indication of increasing poly-crystal cluster formations which, if true. would have significant industrial ramifications.

The nature of the high pressure phase of silica glass under shock compression has long been a mysiery. Shock experiments indicate that a transformation to stishovite should occur, yet there is none to be found in shock-recovered samples. Despite the large temperature variation $\left(<4500^{\circ} \mathrm{K}\right)$, the bulk velocities determined statically to $57 \mathrm{GPa}$ are broadly consistent with the Hugoniot velocities [32]. In the end there probably are several metastable states produced including poorly crystallized states that can be reached depending on the chosen (P-T)-time route.

Superconductivity in high- $T_{c}$ superconductors is generally thought to be dependent on the number of $\mathrm{CuO}_{2}$ layers, $\mathrm{j}$. per chemical unit. A cryogenic DAC four-point resistance measurement on $j=3$ (up to 21 $\mathrm{GPa}$ ) and $\mathrm{j}=4$ (up to $14 \mathrm{GPa}$ ) superconductors reveals that it is the type of $\mathrm{CuO}_{2}$ layer (inner as opposed to outer) that dictates the behavior of $T_{c}(P)$.

Acoustic and thermal DAC measurements on mineral silicates remains extensive $[9,17,34,35,36]$. Conducting ISLS backscattering measurements on gold coaced minerals has proven to be a viable means of attaining shear velocities when none are present in the standard transmission measurements.

\section{ADVANCES IN DAC EXPERIMENTATION}

DAC technology continues to improve with the addition of new cell designs (hydrothermal studies [37], uniaxial stress [38], XRD [39], IR microspectroscopy [40], IR microspectroscopy featuring in situ cryogenic pressure tuning [41], internal ohmic heating [42]) including different gem materials [43], pressure calibration scales (Sm:YAG fluorescence [44], ${ }^{13} \mathrm{C} /{ }^{12} \mathrm{C}$ Raman scale [45], improvements to the ruby fluorescence scale [46]), gas loading devices [47], near hydrostatic pressure medium fluids [48], and simple microlens attachments that effectively triple optical signals[49]. Significant boosts in pressure have been achieved by increasing the slip friction between diamond-metal gasket interfaces [50]. 


\section{CONCLUSIONS AND THE FUTURE}

The information provided above brings together the most significant acoustic DAC studies reported over the last three and a half years. Some of the static thermal measurement property determinations have been highlighted to give one a feel for where things stand and where they are headed. The next three years will bring more $c(P, T)$ studies conducted on simple fluids and fluid mixtures, mineral silicates, energetic materials and refractory metals. Accessible static pressures will increase by at least $30 \%$ and internal heating techniques will be improved upon. The future looks bright!

\section{ACKNOWLEDGMENTS}

The author found the library at LANL to be most accommodating to the task demanded by this paper. This work was partially supported by program CU70 at LANL (Phil Howe) and by the auspices of the U.S. Department of Energy at Lawrence Livermore National Laboratory under contract number W-7405-Eng-48.

\section{REFERENCES}

1. D. A. Boness, J. M. Brown. Shock Waves in Condensed Matter, Presented at the APS Conference on Shock Compression of Matter. Albuquerque. NM., August 14 17. 1989.

2. D. A. Boness, J. M. Brown, and J. W. Shaner, Rarefaction Velocities in Shocked Lead. Presented at the APS Conference on shock Waves in Condensed Matter, Monterey, CA., June 20-23, 1987.

3. H.-K. Mao, R. J. Hemley, Phil. Trans. R. Soc. Lond. A, 354, 1315-32 (1996).

4. V. N. Zharov, T. V. Gudkova, High Pressure Research in Mineral Physics: application to Earth and planetary sciences, Tokyo: Terra, Geophysics Monograph 67, Mineral Phys., 1992, vol. 3, pp. 393-401.

5. J.-P. Poirer, Introduction to the Physics of the Earth Interior, Cambridge University Press, 1991, p. 264.

6. G. Chen, R. Miletich, R. Mueller, H. A. Spetzler, P. E. P. I., 99, 273-87, (1997).

7. O. P. Korobeinichev, L.V. Kuribida A. A. Paletsky, A.G. Shamakov, Combustion Chemistry of Energetic Materials Studied by Probing Mass Spectroscopy, Presented at the Materials Research Society Symposium Proceedings, Boston, MA., Nov. 27-30. 1995.

8. Y.-X. Yan, and K. A. Nelson, J. Chem. Phys., 87, 625765 (1987).

9. E. H. Abramson, J. M. Brown, L. Slutsky, and J. Zaug, J. Geophys. Res.. Vol. 102, B6, 12.253 (1997).

10. L. Brillouin, Ann. Phys., 17, 88 (1922)

II. W. Dollhoph, S. Barry, M. J. Strauss, Presented at the Frontiers of High-Pressure Research Proceedings, 1991.

12. P. S. Peercy, G. A. Samara, B. Morosin, J. Phys. Chem. Solids, 36, 1123 (1975).

13. H. Shimizu, H. Sakoh,, S. Sasaki, J. Phys. Chem, 98, 670 (1994).

14. M. Manga, R. Jeanloz, Geophys. Research Lett., 23, 1845-1848. (1996).

15. G. Shen, H. K. Mao, R. J. Hemley, Laser heating diamond-cell technique: double-sided heating with multimode Nd:YAG laser, Presented at ISAM, Tsukuba, Japan, 1996.

16. G. I. Pangilinan, Y. M. Gupta, J. Appl. Phys., 81, 6662 69, (1997).
17. M. Chai. J. M. Brown. L. Slutsky, Phys. \& Chem. in Minerals, 23. 470-75 (1996)

18. C. Zha, T. S. Duffy, H.-K. Mao, R. J. Hemley, Phys. Rev $B, 48,9246,(1993)$

19. T.S. Duffy, W. L. Vos, C. Zha, R. J. Hemley, H.-K. Mao. Science, 263, 1590-93, (1994)

20. S. Sasaki. N. Nakashima, H. Shimizu, Physica B, 219 \& 220. 380-82, (1996).

21. H. Shimizu. H. Sakoh, S. Sasaki, J. Phys. Chem., 98. 67073. (1994).

22. H. Shimizu, S. Sasaki, Phys. Rev. Lett., 74. 2820-23, (1995).

23. E. H. Abramson, L. J. Slutsky. J. M. Brown. J. Chem. Phys., 100, 4518-26. (1994).

24. S. Sasaki, H. Shimizu. J. Phvs. Sirc. of Japan, 64. 3309 14. (1995).

25. J. M. Zaug, L. J. Slutsky. J. M. Brown. J. Phys. Chem., 98. 6008-16, (1994).

26. K. Weishaupt, H. Krbecek. M. Pietralla. Polymer. 36. 3267-71, (1995).

27. Work in Progress by the author

28. T. P. Russell, G. J. Piermarini, S. Block, P. J. Miller. J. Phys. Chem. 100, 3248-51. (1996)

29. J. K. Rice, T. P. Russell, Chem. Phys. Letr., 234, 195-202, (1995).

30. Y. Nakamura, 1. Fujishiro. T. Tamura, Jpn. Soc. Mech. Eng.. 38, 122-27, (1995).

31. Y. Nakamura, 1. Fujishiro. K. Nishibe, H. Kawakami, J. of Tribology, 117, 519-23. (1995).

32. C. Zha, R. J. Hemley, H.-K. Mao. T. S. Duffy, C. Meade, Physical Rev. B, 50, 13105-12, (1994).

33. D. T. Jover, R. J. Wijngaarden, R. Griessen, E. M Haines, J. L. Tallon, Phys. Rev. B. 54, 10175-85, (1996).

34. C. Zha, T. S. Duffy, R. T. Downs. H.-K. Mao. J. Geophys. Res., 101, 17535.45. (1996).

35. M. Chai, J. M. Brown, L. J. Slutsky, Geophys. Res. Lett. 24, 523-26, (1997).

36. M.Chai. J. M. Brown. L. J.Slutsky. The Elastic Constants of an Aluminous Orthopyroxene to $12.5 \mathrm{GPa}$. J. Geophys. Res., in the press. (1997b).

37. W. A. Bassett, A.H. Shen. M. Bucknum, I.-M. Chou, Rev. Sci. Instrum., 64, 2340-45. (1993).

38. G. Jones, D. J. Dunstan, Rev. Sci. Instrum., 67, 489-93. (1996).

39. D.R. Allan, R. Miletich, R. J. Angel. Rev. Sci. Instrum., 67, 840-42, (1996).

40. J. C. Chervin, B. Canny, J. M. Besson, P. Pruzan, Rev. Sci. Instrum, 66, 2595-98, (1995).

41. R. J. Chen, B. A. Weinstein. Rev. Sci. Instrum., 67, 288389. (1996).

42. S. A. Catledge, Y. K. Vohra. S. T. Weir, J. Akella, J. Phys. Condens. Matter, 9, L67.73, (1997).

43. J.-A. Xu, J. Yen, Y. Wang. E. Huang, Ultrahigh Pressures in Gem Anvil Cells. Amsterdam: Gordon and Breach Science Publishers SA, 1996, pp. 127-34.

44. J. Liu, Y. K. Vohra, Appl. Phys. Lent. 64, 3386-88, (1994).

45. D. Schiferl, M. F. Nicol, J. M. Zaug. S. K. Sharma, T. F. Cooney, S.-Y. Wang, T. R. Anthony, and J. F. Fleischer, The Diamond ${ }^{13} \mathrm{C} / 12 \mathrm{C}$ Isolope Raman Pressure Sensor System for High-Temperature/Pressure Diamond-Anvil Cells with Aqueous and Other Chemically Reactive Samples, (Submitted to, J. Appl. Phys., in the press 1997).

46. M. Chai, J.M. Brown L. J. Slutsky, Geophys. Res. Lett. 23, 3539-42, (1996).

47. T. Yagi, H. Yusa, M. Yamakata, Rev. Sci. Instrum, 67. 2981-84, (1996).

48. D. D. Ragan, D. R. Clarke. D. Schiferl, Rev. Sci. Instrum., 67,494-96. (1996).

49. M. I. Scheerboom, J. A. Schouten. Rev. Sci. Instrum., 67, 853-54, (1996).

50. (J. Akella, S. Weir, private communication). 


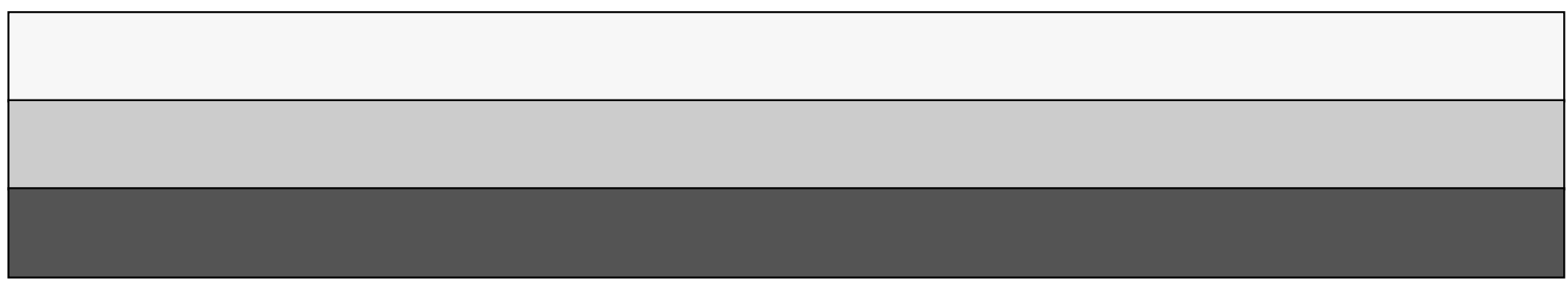

